Éducation

et sociétés

plurilingues
Éducation et sociétés plurilingues

41 | 2016

Varia

\title{
ARED, une expertise au service d'une éducation de qualité au Sénégal
}

Aw Ka Dia

\section{OpenEdition}

Journals

Édition électronique

URL : http://journals.openedition.org/esp/930

DOI : 10.4000/esp.930

ISSN : 2532-0319

Éditeur

Centre d'Information sur l'Éducation Bilingue et Plurilingue

Édition imprimée

Date de publication : 1 décembre 2016

Pagination : 29-42

ISSN : 1127-266X

\section{Référence électronique}

Aw Ka Dia, «ARED, une expertise au service d'une éducation de qualité au Sénégal », Éducation et sociétés plurilingues [En ligne], 41 | 2016, mis en ligne le 20 octobre 2017, consulté le 14 septembre 2020. URL : http://journals.openedition.org/esp/930 


\section{ARED, UNE EXPERTISE AU SERVICE D'UNE ÉDUCATION DE QUALITÉ AU SÉNÉGAL}

Awa Ka Dia

L'uso di un modello bilingue in tempo reale (lingua nazionale e francese) permette agli alunni di acquisire le competenze di base in lettura, matematica e competenze della vita quotidiana fin dall'insegnamento elementare. Gli anni '80 nell'Africa occidentale sono stati particolarmente segnati da una folgorante crescita dell'alfabetizzazione. Creata per sostenere le azioni delle associazioni, l'ONG ARED è in procinto di diventare una struttura di riferimento nell'ambito della realizzazione di materiale didattico per l'educazione bilingue e dell'edizione in lingue africane, in Senegal e nella subregione. Oggi ARED si è collocata fra i leader della produzione di libri in lingue africane grazie a circa 300 titoli realizzati, ad un innegabile professionalismo e una tecnologia all'avanguardia (Macintosh, ultima generazione).

Parole chiave: alfabetizzazione; lingue africane; ARED; Senegal; modello bilingue; insegnamento elementare

Applying a bilingual model in real time to learning a national language + French allows pupils to acquire the basics in reading, maths and daily living skills as of elementary school. The 1980s were particularly important in Senegal due to the rising desire to learn to read and write. Created as a support structure for voluntary associations, ARED, an NGO, is on its way to becoming a reference in the development of pedagogical tools for bilingual education and publishing in the African languages, both in Senegal and the entire region. Today, it is one of the leaders with ca. 300 titles, thanks to its professionalism and the use of advanced technology (Macintosh, latest model).

Key-words: alphabetization; African languages; ARED; Senegal; bilingual model; elementary education

\section{PRÉSENTATION}

Les années 1980 sont particulièrement marquées par une montée fulgurante de l'alphabétisation. En juillet 1987, le congrès organisé par l'Association pour la Renaissance du Pulaar (ARP) avait fait les constats suivants:

- le nombre des individus et des classes engagés dans l'alphabétisation était en train de s'accroître (plus de 500 classes pour le pulaar seulement, dont la plupart fonctionnent grâce au bénévolat);

- le nombre de personnes alphabétisées par les opérateurs de l'époque (société civile, sociétés d'Etat, projets, ONG, associations, etc.) était impressionnant;

- ce qui manquait le plus aux néo-alphabétisés c'était des livres à lire 
ARED, une expertise au service d'une éducation de qualité au Sénégal

A. KA DiA

\section{ARED, DU \\ BÉNÉVOLAT AU PROFESSIONNALI- SME}

pour éviter l'analphabétisme de retour.

- Depuis les années 1980, et même bien avant, l'alphabétisation est devenue un élément incontournable dans les ONG, les projets de développement et les associations à la base. Beaucoup de ressources sont mobilisées pour alphabétiser les populations mais les résultats sont toujours restés en deçà des performances attendues. Car, contrairement à l'opinion selon laquelle il faut des objectifs «fonctionnels» liés au travail ou à la productivité pour pousser les populations à apprendre, la motivation pour s'alphabétiser est plutôt culturelle. Les populations voient en l'alphabétisation une manière de valoriser leur langue, elles voient leur langue comme une manière de valoriser leur culture et elles voient leur langue et leur culture comme le chemin qui peut les mener à un vrai développement (Fagerberg-Diallo 1996).

Fort de ces constats, un groupe composé de 3 linguistes, d'un ingénieur agronome et d'un enseignant de la langue arabe mais tous militants des langues nationales et de la promotion de l'alphabétisation, crée une structure dénommée Groupe d'Initiative pour la Promotion du Livre en Langues Nationales (GIPLLN). L'objectif du GIPLLN était d'éditer et de distribuer des livres en langues nationales. Paradoxalement, dès le départ, les membres du groupe ont renoncé à leurs droits d'auteurs, pour se battre autour d'un même objectif: donner aux néo-alphabétisés de quoi lire.

Cependant, le groupe s'est rendu compte très tôt qu'il faut une structure organisée et professionnelle pour réussir le pari de l'édition en langues africaines.

C'est ainsi que Associates in Research and Education for Development (ARED) est né en 1990 et «se fonde sur les acquis d'une grande association culturelle, l'Association pour la Renaissance du Pulaar (ARP), adossée à un engagement fort de la communauté peulophone» (2).

L'objectif de la création de ARED était d'aider le GIPLLN à être dans des conditions de travail optimales. Alors que ce dernier est constitué surtout d'écrivains et de linguistes, ARED, quant à elle, est composée d'un groupe de jeunes formateurs pour la plupart issus du mouvement associatif capables aussi de faire de l'édition de livres.

Le GIPLLN et ARED ont entrepris alors, au début, la production de livres uniquement, sans jamais prétendre intervenir directement dans les actions d'alphabétisation à la base (animation de classes d'alphabétisation, par exemple). Certains facteurs ont 
ARED, une expertise au service d'une éducation de qualité au Sénégal

A. KA DIA accentué davantage la motivation des deux organisations: les bons manuscrits déjà existants, les néo-alphabétisés qui demandaient des livres pour consolider leurs acquis, et la nouvelle possibilité de production introduite par la $\mathrm{PAO}$.

Etant une ONG, ARED a jugé plus pertinent d'allier production de livres (matériel didactique) et formation. Ainsi, elle se fixe les missions suivantes:

1. Produire une gamme d'ouvrages en langues nationales qui seront mis à la disposition des néo-alphabétisés pour combler le manque de livres enregistré par les néo-alphabétisés au moment où leur effectif s'accroît de manière fulgurante, surtout au début des années 1980.

2. Apporter son appui aux partenaires, dans le domaine de la formation et l'édition en langues nationales. Pour ce faire, des équipes de formateurs sont envoyées sur le terrain pour tester les manuels avant leur impression, recueillir les opinions des formateurs et des moniteurs qui les utilisent.

ARED a opté, dès le départ, de ne pas avoir une zone d'intervention circonscrite. Son choix s'est porté sur le renforcement de capacités des organisations et des acteurs à la base (agents des projets, ONG, volontaires des associations, etc.). Ainsi, au niveau national, ARED appuie les structures partenaires en les formant et en leur fournissant des manuels.

Depuis sa création, ARED a réalisé, avec succès, près de 300 titres publiés dans 7 séries: l'alphabétisation de base (lecture, écriture et calcul); la planification et les aptitudes organisationnelles; la société civile; la culture et le savoir; la santé; l'information scientifique et technique; la religion.

Ces publications sont faites principalement dans six langues nationales (pulaar, wolof, mandinka, sereer, joola, soninke); la plupart des modules de formation visant le renforcement du leadership communautaire sont traduits en français.

Dans l'éducation non formelle, nous cherchons à donner plus de chance aux communautés à travers un renforcement de capacité dans des domaines touchant directement leur vécu quotidien et susceptibles d'aboutir à des changements au niveau communautaire.

Au cours de ces dix dernières années, ARED s'est investie de façon intensive dans la conception, l'expérimentation et la démultiplication d'outils originaux d'animation et de formation sur les questions liées au pastoralisme et au commerce du bétail. ARED ayant compris très tôt que ces problématiques ont une dimension sous-régionale a intégré dans ses démarches la prise en compte de 
ARED, une expertise au service d'une éducation de qualité au Sénégal

A. KA DiA
ARED, DU NON

FORMEL AU FORMEL leur caractère transfrontalier et de l'adaptation des outils aux besoins spécifiques des pays. C'est ce qui lui a valu la sollicitation de nombreuses organisations intervenant à la base au Sénégal et dans la sous-région comme le Mali, le Niger, le Burkina Faso, le Bénin, la Guinée, le Tchad, le Togo, la Mauritanie, etc. Nos équipes ont soit formé des cadres des ministères de ces pays dans les domaines de la recherche et de la planification participative, de la gestion des ressources naturelles, de la gestion des conflits, de l'adaptation au changement climatique, du pastoralisme au Sahel ou du commerce du bétail en Afrique de l'Ouest, soit ont accompagné les ONG et les projets y intervenant dans la formation de noyaux d'animateurs-formateurs capables d'utiliser les outils pédagogiques de ARED pour animer des débats informés au sein de leurs communautés en utilisant leurs langues locales.

C'est dans le souci de favoriser ce partenariat que nous avons développé beaucoup de supports en français car, par principe, ARED ne forme pas sans supports. Pour ce faire, les modules sont d'abord conçus ou traduits ou adaptés en pulaar, avant d'être traduits et adaptés dans leur contexte d'utilisation. Cette approche nous permet de voir les réelles difficultés et les besoins supplémentaires en informations pour les néo-alphabétisés.

Sa volonté de doter les acteurs à la base d'outils fonctionnels bien élaborés et de donner la chance à toute personne alphabétisée dans sa langue de pouvoir trouver de quoi lire ont amené ARED à produire et diffuser plus de 950.000 livres, mais aussi de mener 500 activités de formation pour une appropriation de ces outils. Aujourd'hui plus de 10.000 personnes (animateurs communautaires, formateurs, enseignants, ...) ont été directement formées par ARED au Sénégal, en Afrique et dans le reste du monde. Avec la démultiplication de ces formations, ce sont des centaines de milliers de personnes qui bénéficient des actions de ARED.

L'année 2008, marquée par le retrait des partenaires stratégiques et la disparition de la fondatrice, a été une date repère pour des changements importants opérés au sein de l'organisation.

Après avoir mené une réflexion stratégique approfondie, l'équipe qui a repris le flambeau note que sa mission de «promouvoir une éducation de qualité dans les langues africaines, pour les communautés de base, par la formation, l'édition, les innovations pédagogiques et la recherche-action» a été une réussite dans l'éducation non formelle et que ARED est devenue une structure de référence dans ses domaines d'intervention. Cependant, une analyse de l'environnement de l'éducation au niveau national et interna- 
ARED, une expertise au service d'une éducation de qualité au Sénégal

A. KA DIA

LE PROJET D'APPUI À L'ÉDUCATION DE QUALITÉ EN LANGUES MATERNELLES POUR L'ÉCOLE ÉLÉMENTAIRE AU SÉNÉGAL tional a suggéré la diversification de son partenariat et son offre de services pour continuer à affirmer sa raison d'être et faire face aux défis du futur.

C'est ainsi que ARED décida de s'ouvrir au secteur de l'éducation formelle en proposant au Ministère de l'Education nationale d'apporter son soutien technique dans sa mission de recherche de la qualité dans l'éducation.

Soutenu au départ par la Fondation William and Flora Hewlett et la Fondation Dubai Cares depuis 2014, ARED a tenté d'apporter une réponse à l'amélioration des performances des élèves en lecture/écriture, en mathématiques et en éducation à la science et à la vie sociale (ESVS), à travers un projet expérimental couvrant 208 classes et touchant plus de 10000 élèves des quatre premières années de l'école élémentaire. Cette expérimentation a consisté à mettre en évidence un modèle d'enseignement-apprentissage basé sur les langues premières qui démontre que l'enfant apprend mieux dans sa langue.

Après avoir pris connaissance des résultats catastrophiques issus des évaluations faites sur les enfants en âge de scolarisation, ces adultes de demain qui constituent plus de $40 \%$ de la population subsaharienne, ARED ne pouvait pas rester insensible.

En effet, le Sénégal, à l'instar de l'essentiel des pays de l'Afrique de l'Ouest, est confronté à des problèmes de qualité dans son système éducatif. Beaucoup d'efforts ont été fournis au cours de la dernière décennie pour résoudre les problèmes d'accès, de qualité et de gestion. Les évaluations réalisées dans le cadre du Système national d'évaluation des rendements scolaires (SNERS) ont révélé que «un peu plus de la moitié des élèves maitrisent les compétences de base dans les disciplines fondamentales que sont la lecture, les mathématiques et les compétences de vie courante».

Pour justifier la faiblesse des résultats de l'enseignement, un certain nombre de facteurs ont été identifiés dont:

- La faiblesse de la qualification professionnelle des enseignants;

- L'insuffisance des manuels et matériels didactiques;

- Le manque d'efficacité du dispositif de formation initiale et surtout continue des enseignants;

La non valorisation des langues premières dans le système d'éducation.

Pour pallier à ces manquements, le gouvernement du Sénégal a retenu, entre autres priorités, les suivantes:

- améliorer la qualité des enseignements/apprentissages;

- développer progressivement l'utilisation des langues nationales dans le système éducatif. 
ARED, une expertise au service d'une éducation de qualité au Sénégal

A. KA DIA
Ces deux priorités correspondent aux convictions d'ARED qui considère que l'introduction des langues nationales à l'école élémentaire, combinée avec un bon dispositif de formation, suivi/encadrement et d'une mise à disposition de manuels d'enseignement/apprentissage peut aider à résoudre les problèmes de qualité auxquels est confronté le système éducatif sénégalais.

L'intervention officielle des organisations non gouvernementales dans le système formel au Sénégal n'est pas aussi simple qu'on le pense. Malgré sa volonté de soutenir l'Etat dans sa recherche de qualité, ARED a fait face à des obstacles. En 2009, lorsque ARED s'est approchée des autorités éducatives pour obtenir l'autorisation d'entrer dans les classes pour démarrer l'expérimentation, elle a rencontré une opposition formelle qui l'a obligée à organiser les cours pendant les après-midi libres (lundi, mercredi, vendredi) avec les arguments suivants:

- il ne faut pas perturber le curriculum de l'éducation de base qui est en cours de généralisation;

- le bilan de l'expérimentation de la mise à l'essai de l'introduction des langues nationales conduite par l'Etat de 2002 à 2008 n'est pas encore tiré;

- l'Etat n'a pas encore pris des dispositions officielles pour relancer sa politique d'introduction des langues nationales à l'école.

Cette organisation des cours en dehors des heures normales a créé beaucoup de perturbations sur le déroulement de l'expérimentation: les maîtres expérimentateurs ont réclamé des indemnités en compensation à la surcharge de travail, d'autant plus que l'Etat les indemnise pour toute activité expérimentale ou spéciale; certains enfants n'étaient pas disponibles les après-midi du fait des études coraniques ou d'autres occupations habituelles; certains acteurs à la base considéraient l'expérimentation comme une action privée parce que se déroulant en marge du programme officiel.

Malgré toutes ces difficultés, ARED a su mobiliser les acteurs de la base au sommet pour mener à bien son action. Les maîtres, les directeurs, les inspecteurs et même les agents des directions techniques nationales ont été impliqués dans le développement du matériel didactique, de la formation des enseignants et du suivi des apprentissages.

Le bilan sans équivoque réalisé au bout de deux ans a créé une unanimité autour des résultats positifs apportés par l'utilisation des langues nationales pour renforcer le niveau des élèves. Les enseignants et les inspecteurs ont relevé, avec preuves à l'appui, devant les décideurs du système, les effets positifs de l'intervention 
ARED, une expertise au service d'une éducation de qualité au Sénégal

A. KA DIA

\section{ARED, DE LA PÉRIPHÉRIE AU CEUR DU PROGRAMME OFFICIEL}

\section{L'EXPÉRIENCE D'ARED DANS L'ENSEIGNEMENT BILINGUE}

\section{BILAN DES ACTIVITÉS}

d'ARED sur le niveau d'implication des services du Ministère dans le pilotage, l'adhésion des communautés, l'amélioration de la pratique de classe des enseignants, les performances des élèves à travers des évaluations harmonisées. C'est ce qui a permis à ARED d'enregistrer son premier succès, celui d'amener les autorités à reconnaître la qualité de son programme et à l'autoriser de l'introduire dans les horaires officiels.

Ayant vite compris que tout nouveau changement est difficile, ARED a pris l'option de convaincre par la preuve et de faire un plaidoyer par l'action et l'implication des acteurs clés.

Ainsi, après avoir eu l'autorisation, ARED a décidé d'adopter une démarche progressive et prudente d'introduction de son programme dans les horaires officiels.

Cette expérimentation bilingue s'est déroulée en deux phases.

Première phase (2009-2011): cours pendant les après-midi libres. Cette première phase a été réalisée en deux étapes:

1. Une étape de «mise à l'essai» qui s'est déroulée de novembre 2009 à juin 2010. Elle a permis d'élaborer les guides pour les enseignants et les manuels de la première année en pulaar et en wolof, de les tester dans quatre classes de 1 ère année, de les finaliser et de les imprimer en quantité suffisante pour l'expérimentation.

2. Une étape de «mise en expérimentation» qui s'est déroulée d'octobre 2010 à juin 2011. Elle a consisté à la mise en œuvre du modèle d'enseignement-apprentissage dans 30 classes pulaar et wolof réparties dans les régions de Dakar, Saint-Louis et Kaolack, avec toutes les dispositions pédagogiques et organisationnelles nécessaires.

Au bout de cette étape, un bilan de l'expérience avec les trente classes est fait.

Il a montré des points positifs importants:

-Un bon partenariat entre ARED et le Ministère;

-Un pilotage dynamique et concerté;

-Une bonne implication des services déconcentrés et de l'encadrement rapproché dans la formation des enseignants et le suivi des classes;

-Une adhésion des communautés;

-Une amélioration de la pratique de classe des enseignants;

-Des impacts positifs sur les performances des élèves notés à travers des évaluations harmonisées;

-Un matériel didactique pour le CI (première année de l'école élémentaire) conçu et testé.

Cependant, la mise en œuvre de l'expérimentation s'est heurtée à 
ARED, une expertise au service d'une éducation de qualité au Sénégal

A. KA DIA

\section{CONSÉQUENCES:}

un certain nombre de difficultés:

\section{Cours tenus en dehors des horaires normaux}

Le Sénégal s'est doté d'un curriculum de l'éducation de base qui est mis en expérimentation avant que sa généralisation ne commence en 2009. C'est pourquoi les autorités étaient réticentes, au départ, à autoriser une nouvelle expérimentation. Cependant, une petite ouverture est donnée à ARED, c'est à dire faire les cours les après-midi libres (lundi, mercredi, vendredi) pour ne pas perturber le curriculum de l'éducation de base. Là, ils prenaient l'allure de cours de renforcement.

Comme déjà idiqué, les maîtres expérimentateurs ont réclamé des indemnités pour compenser la surcharge de travail, d'autant plus que l'État les indemnise pour toute activité expérimentale ou spéciale;

certains enfants n'étaient pas disponibles les après-midi du fait des études coraniques ou d'autres occupations familiales;

certains acteurs à la base considéraient l'expérimentation comme une action non officielle parce que se déroulant en marge du programme et de l'emploi du temps officiel.

Un retard dans le choix définitif des écoles et maîtres

Les écoles et les maitres n'ont été choisis qu'après l'ouverture des classes. Les cours n'ont démarré qu'à partir de janvier. Cela a posé des problèmes dans la gestion des apprentissages des deux langues (français et langue nationale) et dans la détermination de la valeur ajoutée de la langue nationale par rapport au français.

Un non respect des critères de choix des maîtres expérimentateurs dans quelques endroits

Dans certaines écoles, les maitres expérimentateurs ne maitrisent pas la langue nationale d'enseignement. Cela a occasionné le recrutement d'assistants communautaires pour mener à bien l'expérimentation.

Au regard des acquis et des difficultés notés, l'atelier a validé la proposition d'ARED, qui consiste à reprendre l'expérimentation dans les conditions définies de façon participative avec les partenaires. Ainsi les stratégies suivantes ont été proposées:

-intégrer le programme dans l'emploi du temps officiel;

-consolider la collaboration avec les trois IA (Dakar, Kaolack, Saint-Louis);

-poursuivre le travail dans les deux langues (pulaar et wolof);

-mettre à profit les acquis de l'expérimentation faite dans les 30 classes.

En août 2011, le Ministère a organisé un atelier pour harmoniser 
ARED, une expertise au service d'une éducation de qualité au Sénégal

A. KA DIA

DEUXIÈME PHASE (2011-2013): COURS DANS LES HORAIRES OFFICIELS

\section{ACTEURS CLÉS DU PROGRAMME}

\section{LE PILOTAGE DU PROJET}

les interventions des acteurs dans le cadre de l'Enseignement bilingue. Cet atelier a formulé d'importantes recommandations allant dans le sens du respect des approches et normes fixées par le Curriculum de l'Éducation de Base.

Pour se conformer à toutes ces exigences, l'ONG ARED a soumis au Ministère un programme expérimental redimensionné de 14 classes (08 CI et 06 CP)_en 2011-2012 avec l'intégration des cours en langue nationale dans l'emploi du temps officiel.

Les résultats satisfaisants obtenus ont permis l'extension, en 20122013, à cent nouvelles classes bilingues et couvre, outre le domaine Mathématique, l'ensemble du domaine Langue et Communication.

Aujourd'hui, le programme couvre 208 classes pour 10593 enfants (entre 6 et 10 ans). L'apprentissage démarre en langue nationale et les acquis sont transférés au français, langue seconde, et le gain de temps est utilisé pour renforcer et approfondir le degré de maitrise des apprentissages.

Dans une perspective de mise à l'échelle, ARED a impliqué, dès le départ, les structures de l'Etat, à tous les niveaux pour faciliter l'appropriation du modèle. Ainsi, les cours sont tenus par les enseignants/fonctionnaires de l'Etat et tout le travail d'organisation, d'implantation, de formation des enseignants, de suivi et encadrement, d'évaluation et même de pilotage a été réalisé dans un partenariat étroit avec le Ministère de l'éducation nationale.

Pour assurer un ancrage communautaire, un important travail de sensibilisation a été conduit au niveau des communautés, en collaboration étroite avec les inspections et les directions techniques du Ministère. Les comités de gestion des écoles (CGE) ont été également formés pour une meilleure prise en compte du bilinguisme.

Le programme d'ARED est mis en ouvre en partenariat avec les structures techniques spécialisées du MEN. Les services les plus impliqués dans l'exécution du programme sont l'Equipe Technique de Pilotage (ETP) qui regroupe les représentants de ARED et de diverses directions. Ses missions se résument à l'orientation stratégique, au pilotage, aux formations, aux évaluations, à la validation des outils pédagogiques et au suivi du suivi local.

L'Inspecteur d'Académie (IA) est chargé de consolider les rapports produits par les IEF (Inspecteurs de l'éducation et de la formation) en un rapport trimestriel régional à transmettre à ARED. ARED a transmis à chaque IA un modèle de fiche de synthèse trimestrielle. 
ARED, une expertise au service d'une éducation de qualité au Sénégal

A. KA DIA

\section{AU NIVEAU DES ÉCOLES}

\section{LES SPÉCIFICITÉS} DU PROGRAMME

LES RÉALISATIONS DU PROGRAMME
Les IEF sont responsables de la coordination, de la mise en œuvre et du suivi des activités dans leurs circonscriptions. Ils sont chargés de choisir les écoles et les maitres, d'organiser la formation des enseignants, de suivre et d'assurer l'encadrement rapproché des directeurs et enseignants, et la mobilisation des communautés autour de l'école et des enseignants. Ils produisent des rapports mensuels sur la base d'une fiche de suivi/synthèse qu'ils transmettent à l'IA.

Les directeurs d'écoles assurent un encadrement des maîtres qui sont dans leur école et attestent de l'effectivité du fonctionnement des classes. Ils sont tenus de soumettre un rapport mensuel aux IEF sur le déroulement des cours. Ils sont membres des CGE, c'est-à-dire des comités de gestion des écoles, chargés de veiller aux conditions matérielles de la mise en œuvre du programme dans les écoles, à la ponctualité et à l'assiduité des maitres et des élèves.

L'utilisation d'un modèle bilingue en temps réel - langue nationale et français dès les premiers jours de la première année d'enseignement/apprentissage - permet aux élèves de l'école primaire d'acquérir rapidement les compétences de base en lecture, mathématique et compétences de la vie courante dès les quatre premières années de l'enseignement élémentaire. Ce modèle bilingue à temps réel s'appuie sur:

1. l'existence d'un programme bilingue pour les quatre premières années de l'enseignement élémentaire.

2. une formation efficace des enseignants.

3. Une mise à disposition suffisante et à temps du matériel didactique adéquat aux enseignants et aux élèves.

Grâce à la démarche de recherche-action adoptée dès le début et de partenariat avec des experts, ARED a construit son modèle bilingue conformément au curriculum de l'éducation de base (CEB) et aux référentiels de compétences y afférant.

Des experts de l'Université appuient le processus de réalisation des manuels et guides en mettant à disposition l'expertise et les informations sur le bilinguisme et ses principes. Les experts interviennent pour évaluer et garantir les normes scientifiques du contenu.

Depuis le début du programme, ARED s'est fixé comme objectif de mettre à la disposition des élèves et des enseignants, directeurs d'école et inspecteurs les supports pédagogiques nécessaires. Chaque élève est doté de la première à la quatrième année, d'un 
ARED, une expertise au service d'une éducation de qualité au Sénégal

A. KA DIA manuel de lecture et de mathématique en langue nationale, d'un manuel de lecture en français, d'un manuel d'ESVS, d'un cahier d'exercice et d'autres livres de lecture pour le plaisir. Les enseignants, les directeurs et les inspecteurs disposent, chacun, d'un référentiel de compétences bilingue, d'un guide lecture, d'un guide de mathématique et d'un guide terminologique bilingue. ARED a mis également à leur disposition un manuel de grammaire en langue nationale, ainsi que le décret organisant la transcription dans la langue nationale.

Dans le cadre du partenariat avec le Ministère de l'Education nationale, ARED a contribué à la finalisation du manuel ESVS pour le CI de ELAN.

ARED dispose d'un plan de formation initiale et continue des personnels en charge de l'enseignement bilingue validé par les parties prenantes du programme. Il épouse les principes du Ministère de l'Education Nationale en la matière.

Le plan de formation s'inscrit dans une dynamique de transfert de la formation initiale aux CRFPE. Dans la stratégie de formation des personnels du Ministère de l'Education Nationale (MEN), il est implanté dans chaque Inspection d'Académie du pays, un centre régional de formation appelé Centre Régional de Formation des Personnels de l'Education (CRFPE). Toutes les formations initiales des enseignants (éducateurs du préscolaire, enseignants du cycle élémentaire,...) sont réalisées dans ces structures. En effet, les maîtres nouvellement désignés par les IEF pour tenir les nouvelles classes de CI ont été regroupés dans 3 CRFPE (Rufisque, Kaolack et Saint Louis) pour recevoir une formation initiale sur le bilinguisme au mois de septembre 2014 pendant 13 jours.

Par contre, la formation continue (renforcement des capacités du personnel enseignant) est faite au niveau des Inspections de l'Education et de la Formation (IEF). En effet, la proximité des IEF avec les enseignants relevant de leurs circonscriptions scolaires leur permet de déceler les besoins des enseignants et ainsi de cibler leur formation. Les missions de suivi des classes effectuées sur le terrain par ARED et les agents du MEN regroupés dans l'ETP a pour objectifs de recenser les difficultés des enseignants, notamment celles liées à la pratique de classes bilingues (planification des apprentissages, élaboration de fiches de préparation à la lumière du bilinguisme, tenue de documents de gestion de classe bilingue, déroulement des contenus, etc...).

Depuis 2014, ARED est dotée d'un plan de suivi évaluation spécifiant les types d'informations que les encadreurs (directeurs, ins- 
ARED, une expertise au service d'une éducation de qualité au Sénégal

A. KA DiA pecteurs, ARED, ETP) doivent produire.

Le Suivi-Evaluation (S\&E) se focalisera essentiellement sur:

1) le processus de renforcement des capacités des enseignants et de leurs aptitudes à tenir efficacement les classes bilingues;

2) les capacités des directeurs et inspecteurs à encadrer les enseignants et à remonter les informations;

3) les capacités et l'engagement des communautés à accompagner la mise en œuvre du modèle dans les écoles. Tout cela concourt à la réalisation de l'impact qui sera mesuré à travers le niveau d'amélioration des compétences des élèves.

Le dispositif de S\&E intègre le système mis en place par l'Etat du Sénégal et qui donne des responsabilités aux directeurs, inspecteurs de circonscriptions, inspecteurs d'académie, directions nationales et même des communautés à travers les CGE dans le suivi des processus d'enseignement-apprentissage et de leur impact. En effet, ARED identifie les rôles et outils complémentaires qui permettent de prendre en charge les spécificités de l'enseignement bilingue.

Les IEF organisent des évaluations tous les 3 mois sur décision de l'IA. Dans certaines IEF, elles sont standardisées et se caractérisent par l'administration, pour chaque niveau, d'un même type d'épreuves, avec un même crédit horaire pendant une période déterminée. Autrement dit, les conditions d'administration sont identiques pour l'ensemble des élèves d'une circonscription éducative. Avant leur mise en œuvre, une commission organisée par l'IEF fait l'état des lieux pour déterminer les niveaux de progressions. Dans d'autres, ce sont des «compositions». Elles sont aussi des évaluations trimestrielles, mais qui se déroulent au niveau de l'école sous la supervision des IEF. Chaque école propose ses épreuves, en fonction de ses progressions dans les différentes disciplines.

L'Outil de suivi des performances classes bilingues ARED est conçu et validé avec tous les inspecteurs pour que les directeurs y reportent les résultats des élèves de leurs écoles, aussi bien pour les classes bilingues que monolingues.

\section{Des évaluations externes indépendantes}

«L'évaluation du programme bilingue de l'ONG ARED confirme que le bilinguisme est une composante importante dans l'amélioration de la performance des élèves en lecture et en mathématiques dans l'enseignement primaire au Sénégal. L'évaluation démontre d'ailleurs que parmi tous les critères étudiés, le bilinguisme tel que mis en œuvre dans le cadre du programme ARED a l'effet le plus positif sur les compétences des élèves, suivi de la disponibilité des manuels, du profil des maitres/directeurs (genre, 
ARED, une expertise au service d'une éducation de qualité au Sénégal

A. KA DIA expérience, méthodologie d'enseignement), du soutien de l'équipe technique pédagogique et de l'implication du comité de gestion dans la vie de l'école" (Résumé 2014).

En outre, cette évaluation montre que «Les scores du groupe expérimental sont à tous les niveaux supérieurs à ceux du groupe témoin» (1).

\section{Graphigue 3: Comparaison entre le seuil de maitrise et les scores globaux pour le groupe expérimental et le groupe témoin Scores Globaux de Lecture en Juin

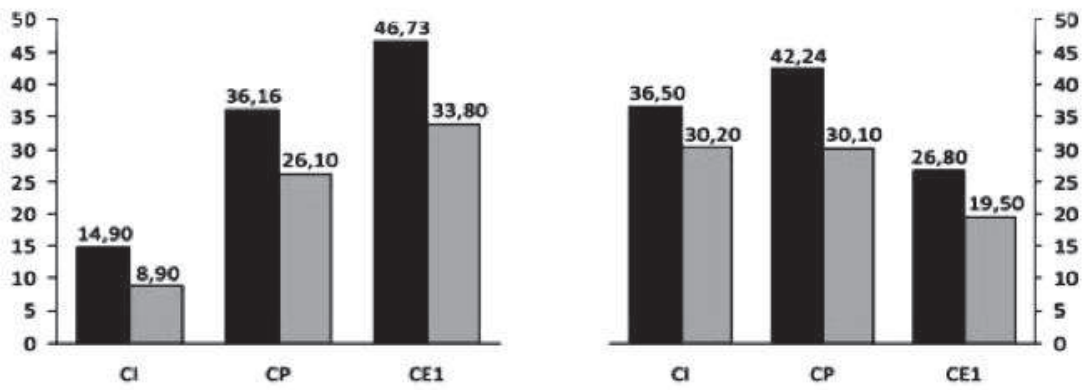

Groupe Experimental $\square$ Groupe Temoin

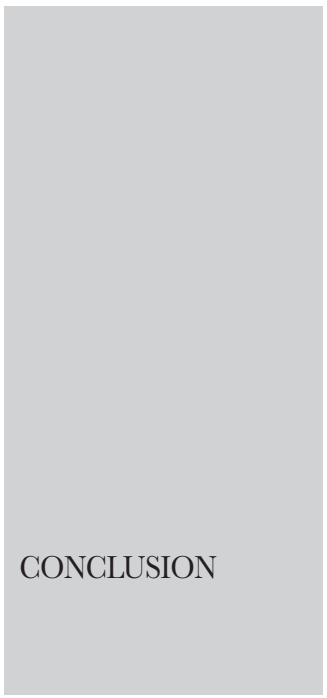

(Cf Résumé du Rapport d'évaluation d'impact du modèle ARED, Dalberg, Janvier 2014)

\section{Implication des autorités éducatives}

Depuis le début, ARED a privilégié l'implication des autorités du MEN: réflexion, sensibilisation, mise en œuvre du programme, suivi, évaluation, etc.

\section{Adhésion des communautés}

Chaque école a un comité de gestion opérationnel (Comité de Gestion de l'Ecole, CGE) mandaté pour mettre en œuvre toute initiative éducative tant qu'elle est en conformité avec le programme scolaire national. Dans le programme en cours, ARED a eu à former les comités de gestion, à deux reprises, à travers des sessions de formation.

Créée pour soutenir les actions d'alphabétisation des associations, ARED est en passe de devenir une structure de référence dans le domaine du développement du matériel didactique et de l'édition en langues africaines au Sénégal et dans la sous-région. 
ARED, une expertise au service d'une éducation de qualité au Sénégal

A. KA DiA

\section{RÉFÉRENCES}

NOTE
Aujourd'hui, ARED s'est positionnée parmi les leaders de la production de livres en langues africaines avec environ 300 titres réalisés, grâce à un professionnalisme indéniable et une technologie de pointe (Macintosh, dernière génération).

Les modules de formation d'ARED, fruits d'une recherche-action soutenue, ont franchi les frontières du Sénégal pour être adaptés dans d'autres contextes à d'autres langues pour servir, en fin de compte, d'outils préférés aux organisations de la sous-région: Mali, Burkina Faso, Bénin, Niger, Tchad, etc.

Cependant, certains défis sont encore à relever. Il s'agit, en plus de l'épineux problème de la distribution, d'une édition dans les langues transfrontalières (fulfulde, bamanakan, etc.) pouvant être utilisée par plusieurs pays partageant une même langue.

FAGERBERG-DIALLO S. 1995. L'éducation et la lecture en langue pulaar: Le cas du Sénégal, revue EAD, Education des Adultes et Développement.

LY M. A. 2015. Recherche-action au Sénégal pour la formation des formateurs, Institut de l'UNESCO pour l'apprentissage tout au long de la vie (UIL).

Résumé du Rapport Evaluation du modèle bilingue ARED. 2014. Dalberg-Dakar (janvier).

(1) Le groupe témoin est constitué de classes monolingues qui ont les mêmes caractéristiques que les classes expérimentales et qui sont choisies pour permettre une comparaison. 